\title{
Examining the effects of bodygrip 330 traps on domestic cat (Felis catus) cadavers
}

\author{
Rebecca Kagan' DVM DACVP, Jessica Elbert² DVM, Max Juriga³ DVM
}

\begin{abstract}
Bodygrip traps (also called Conibear traps) are nontoothed kill traps used for furbearers and nuisance wildlife. These traps pose a danger to nontarget animals, such as protected wildlife, domestic animals, and people, more so when used on dry land. Because of the potential for intentional or accidental misuse, diagnosis of bodygrip trap injuries may be of consideration in forensic casework. To determine whether trap-related injuries can be identified to narrow down or confirm the cause of death, standard 330 and Magnum 330 bodygrip traps were used on cadavers of 9 domestic cats (F. catus). Trap jaws were engaged in various locations on the bodies to simulate potential live entrapment situations. The cadavers were then imaged and necropsied. Trap-related damage was present only in one (1/9) cat and consisted of liver fractures, likely augmented by the presence of a full stomach. The remaining cats $(8 / 9)$ had no grossly or radiographically visible injuries. Findings are consistent with past live animal studies in which trap-related injury was only rarely observed or documented. Because no specific injuries can be attributed to bodygrip traps, the diagnosis must rely on the circumstance and rule out other likely causes of death.
\end{abstract}

KEYWORDS: veterinary forensic sciences, pathology, cause of death, wildlife, domestic cat, Felis catus, trap, body grip, bodygrip

Bodygrip traps (also called Conibear traps) are nontoothed kill traps used on dry land and underwater for a variety of small to midsized furbearers. These traps were developed in the 1920s as a "humane," efficiently-deadly alternative to other trapping methods. They have been commercially available since the 1950 s.

During the 2014-2015 trapping season, approximately 106,000 licensed trappers in the United States reported using bodygrip traps for their primary target species (Responsive Management 2015). Statistics regarding the bycatch of domestic animals from legal and illegal bodygrip trapping are not collected. Born Free USA maintains a database of nontarget trapping incidents, but reporting is voluntary (Non-target ... 1990-). According to the database, 131 incidents involving kill-type traps have been reported in dogs and cats in the United States and Canada between 1992 and 2019. Of the reported outcomes, trapping incidents resulted in the deaths of 85 dogs and 9 cats and non-fatal injuries in 21 dogs and 7 cats. Additionally, 1 dog was released without injuries, and 8 canine trapping incidents did not have a reported outcome.
As this database relies on voluntary public reporting, it is reasonable to assume that actual numbers of injury and death in domestic animals is underestimated.

Bodygrip traps have smooth, square rotating jaws. When activated, springs on either side cause the jaws to rapidly and forcefully close (FIGURE 1) along 1 or 2 transverse planes, depending on the size of the animal and its position when the trigger is activated. In the intended scenario, 1 of the 2 sets of jaws compresses the neck, cutting off blood flow and resulting in rapid loss of consciousness and death. Loss of consciousness within 300 seconds (5 minutes) in $70 \%$ of animals is sufficient to meet U.S. animal welfare standards for traps set on land (AFWA 2017). Unfortunately, inherent design flaws have contributed to the variable efficacy of bodygrip traps. Also, morbidity and mortality of nontarget species can be high (Barrett et al. 1989; Naylor and Novak 1994).

Two types of bodygrip 330 traps are commercially available: the standard trap and the Magnum trap. The latter is marketed as having trap jaws that close tighter than the

\footnotetext{
1 U.S. Fish and Wildlife Service National Forensics Laboratory, 1490 East Main Street, Ashland, OR 97520-1310 USA

2 University of Georgia College of Veterinary Medicine, Department of Pathology, 501 D. W. Brooks Drive, Athens, GA 30602 USA

3 Virginia-Maryland College of Veterinary Medicine, 205 Duck Pond Drive, Blacksburg, Virginia 24061 USA

Corresponding author: Rebecca Kagan, rebecca kagan@fws.gov

Received 2019 Jan 24; revised 2019 Dec 9; accepted 2020 Jan 3.
} 
standard type. The 330 traps are the largest sized bodygrip traps.

Lesions associated with unmodified body traps are poorly documented. The few completed studies have demonstrated a distinct lack of gross or histologic lesions (Barrett et al. 1989; Proulx et al. 1995). The purpose of this study was to determine whether by-catch of domestic cats with a bodygrip 330 trap would result in any distinct fracture patterns or soft tissue injury that could be identified after the immediate postmortem period.

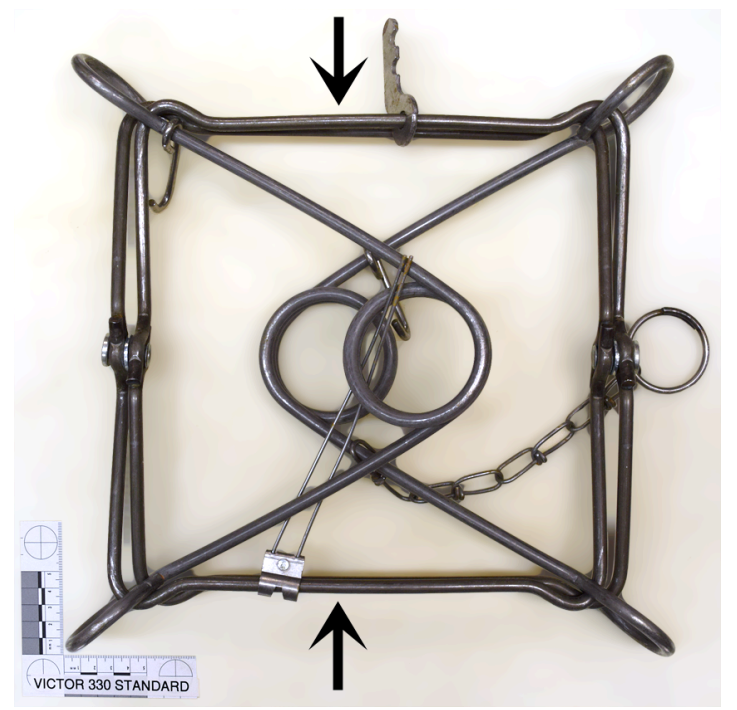

FIGURE 1-An example of an unset bodygrip 330 trap.

The jaw ends are at the top and bottom of the photo (arrows).

\section{Materials and Methods}

Two commercially available bodygrip traps were obtained: an Oneida Victor Conibear 330-2 standard trap and a Duke 330 Magnum trap. The standard trap was certified to meet international humane trapping standards, as defined in the Canadian Competent Authorities Trap Certification Procedures and Protocol, for bobcat (F. rufus), beaver (Castor canadensis), North American river otter (Lutra canadensis) and the Canada lynx (Lynx canadensis).

Both traps had a 4-way trigger with a 10-inch jaw spread. The actual dimensions of the standard and Magnum traps used in this study were $10.5 \times 11.5$ inches and $9.3 \times$ 11.3 inches, respectively. The gaps between the closed jaws, measured with a caliper at the midpoint, were $4.5 \mathrm{~mm}$ for the standard trap and $6.0 \mathrm{~mm}$ for the Magnum trap (FIGURE 2).

Nine adult domestic cat cadavers were donated to the U.S. Fish and Wildlife Service National Forensics Laboratory by a local animal shelter. The cats were euthanized for reasons unrelated to this study and received fresh on the day they were euthanized. The weight, crownto-rump length, head, and thoracic circumferences were
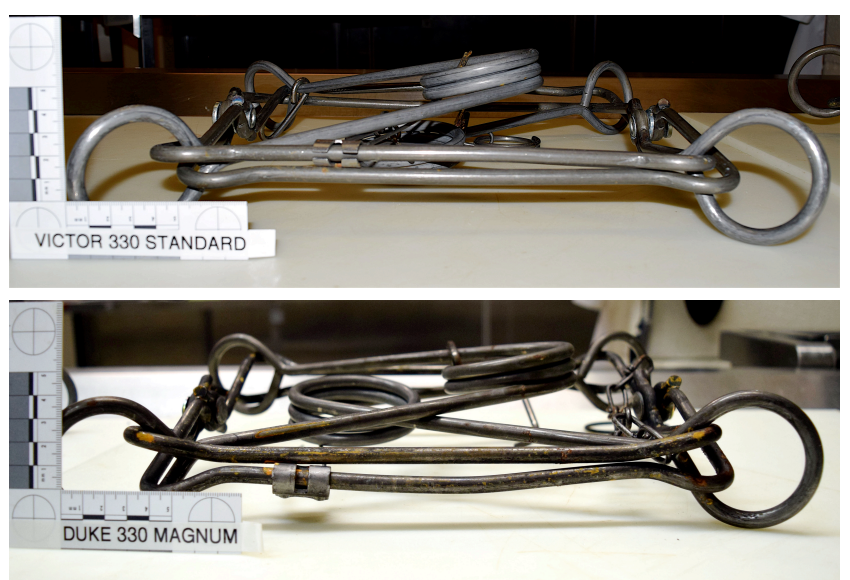

FIGURE 2-Jaw gaps. The gap in the closed jaws, measured at the midpoint, of the standard bodygrip 330 trap (top) was $4.5 \mathrm{~mm}$; the Magnum bodygrip 330 trap (bottom), $6.0 \mathrm{~mm}$.

recorded. Full-body, orthogonal radiographs were taken of each cat at the beginning of the study. There were no radiographic abnormalities detected.

No selection criteria were established. Cats were randomly designated to study groups. Four cats (designated $1,2,3,4)$, all intact adult males, were used to evaluate the standard trap. These cats were stored at $40{ }^{\circ} \mathrm{F}$ for 4 days prior to use, which resulted in mild abdominal bloat that was relieved via an 18-gauge needle inserted before further study. Five cats were used to evaluate the Magnum trap: 1 neutered male $(1 \mathrm{M}), 2$ intact females $(2 \mathrm{M}, 3 \mathrm{M})$, and 2 intact males $(4 \mathrm{M}, 5 \mathrm{M})$. All analyses were done on the same day that these cats were euthanized.

Heavy-duty setting tongs were used to set the traps. Each cat was placed in a set trap to mimic points at which contact with the trigger might be made in a live situation (FIGURE 3). The trap was sprung by pulling on a piece of string that was loosely taped to the trigger.

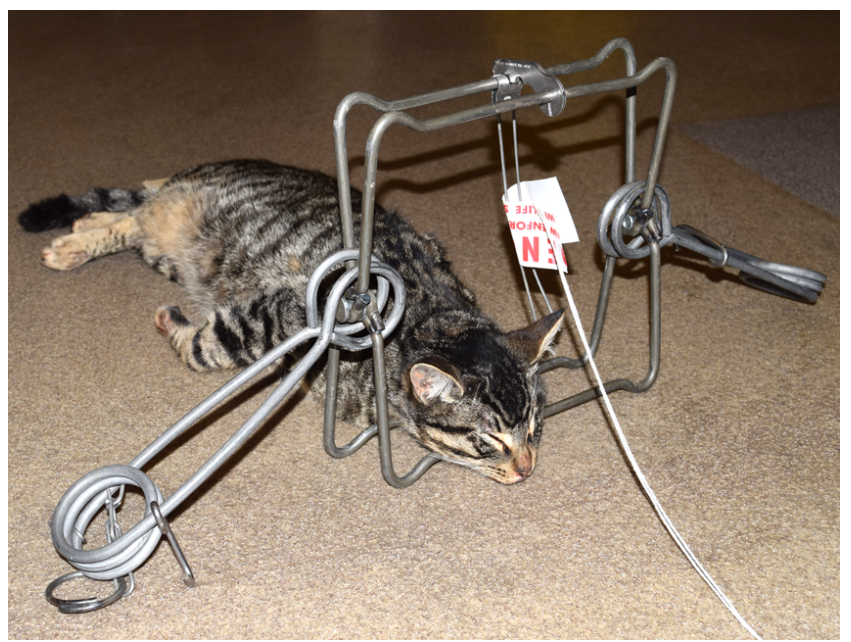

FIGURE 3-Placement of a cat cadaver (cat 2) in a set bodygrip trap. A piece of string taped to the trigger enables the trap to be sprung from a distance. 
Cats were trapped in various positions on the body cavity, head, neck, and limbs along 1 or 2 transverse planes (FIGURE 4). After the trap was removed from each cat, second sets of full-body, orthogonal radiographs were taken. Computed tomography scans (Animage Fidex) of cats 2 and $3 \mathrm{M}$ were made. A gross necropsy was then performed on each cat. The necropsy included complete removal of the skin as well as the removal and gross examination of each organ. The skulls were left intact.
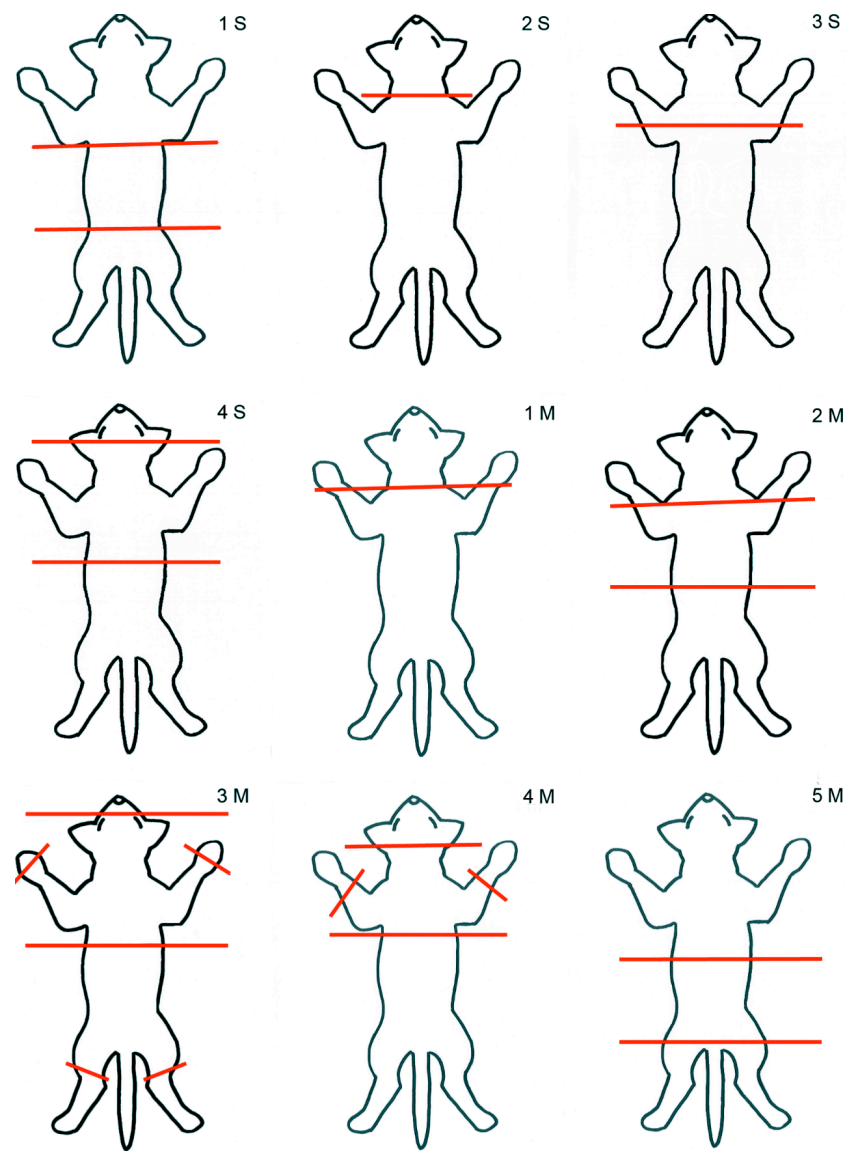

FIGURE 4-Positions of sprung bodygrip trap jaws (red lines) on 9 cat cadavers. $\mathrm{S}$, standard trap. M, Magnum trap.

\section{Results}

\section{Standard bodygrip 330 trap}

Despite refrigeration, there were changes due to early decomposition in the form of intestinal bloat, subcutaneous gas accumulation, and leakage of decomposition (purge) fluid through the oral cavity. No other external or radiographic abnormalities were present before or after entrapment. Entrapment did not cause any visible soft tissue tears, despite early softening due to decomposition. There were no fractures or other trap-related, postmortem injuries observed on gross examination or imaging studies.

\section{Magnum bodygrip 330 trap}

Only one cat had any grossly visible changes that could be attributed to the trap. Cat $3 \mathrm{M}$ had multiple liver fractures at the hilus (FIGURE 5). This cat also had a full stomach. No postmortem injuries were identified on any of the radiographs.

\section{Discussion}

Bodygrip trap closure on areas along the length of the body cavity, neck, head, and limbs did not cause any visible injuries when the standard trap was used and only in one instance with the Magnum trap. Because the jaw gap of the closed Magnum trap is not less than that of the standard trap (in fact, the gap is wider in this particular Magnum trap), this injury likely is not attributable to the Magnum type's purported enhancements. It is more likely that the full stomach of this cat caused additional compression of the liver, which resulted in tears. Another cat that was trapped in the same location but had an empty stomach (cat 4) did not have liver damage. Though more subtle signs of acute to sub-acute soft tissue trauma in the form of hemorrhage or pulmonary edema cannot be assessed in a cadaver study; overall, findings are consistent with those in published live animal studies (Barrett et al. 1989; Proulx and Barrett 1993; Proulx et al. 1995).

Bodygrip traps work primarily by rapidly and forcefully compressing soft tissues, like a giant mousetrap, to impede respiration, blood flow, or both, depending on the location of the trap jaws on the body. Despite the force with which the traps close, the jaws are designed to have a small gap when closed to prevent inadvertent crushing of human fingers (AFWA 2017). Unfortunately, this leads to a

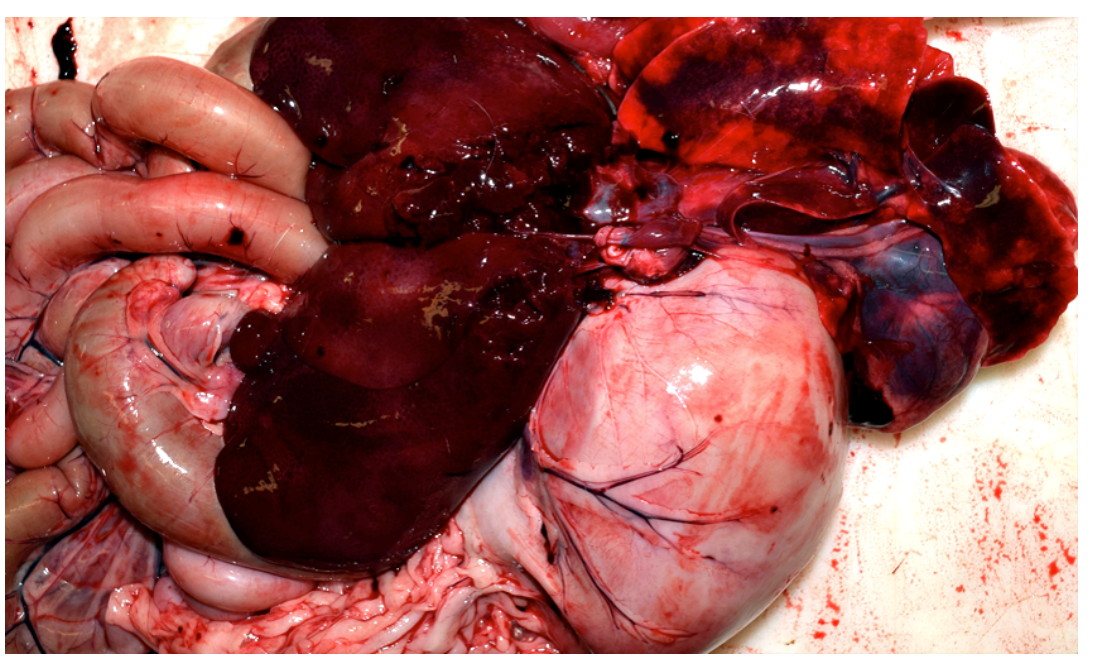

FIGURE 5-Cat 4M. Liver fractures were caused by the combined effects of the Magnum trap jaws and a full stomach. 
reduction in killing efficiency. The jaw gap, even with a Magnum-type trap, along with a spring strength that is not sufficient to break bones, reduces the likelihood of gross lesions.

\section{Conclusions}

Diagnosing a bodygrip trap as a cause of death would be difficult without knowledge of a corroborating circumstance (e.g., the animal is found dead in or near a trap). It should be noted that traps altered to eliminate the jaw gap have been shown to cause grossly visible injuries, such as cervical and cranial fractures in marten (Martes americana) and lynx (Barrett et al. 1989; Proulx et al. 1995). In these cases, fractures may provide supportive evidence of a bodygrip trap as the cause of death.

Future studies could involve further data collection from live-trapping incidents in domestic dogs and cats.

\section{Disclosures}

The findings and opinions contained herein are those of the author and do not necessarily reflect the views of the U.S. Fish and Wildlife Service. The authors received no financial support for the research, authorship, or publication of this article.

\section{References}

(AFWA) Association of Fish and Wildlife Agencies, Furbearer Conservation Technical Work Group (Washington, DC). 2017. Bodygrip traps on dryland: a guide to responsible use. Washington (DC): AFWA (US)

Barrett MW, Proulx G, Hobson D, Nelson D, Nolan J. 1989. Field evaluation of the C120 Magnum trap for marten. Wildl Soc Bull. 17:299-306. jistor.org/stable/3782389.

Naylor BJ, Novak M. 1994. Catch efficiency and selectivity of various traps and sets used for capturing American martens. Wildl Soc Bull. 22:48996. jstor.org/stable/3783393.

Non-target trapping incidents database. 1990-. Silver Spring (MD): Born Free USA. [accessed 2018 Mar 7, 2020 May 3]. bornfreeusa.org/ trapping-incidents-search.

Proulx G, Barrett MW. 1993. Evaluation of mechanically improved Conibear $220^{\text {mt }}$ traps to quickly kill fisher (Martes pennanti) in simulated natural environments. J Wildl Dis. 29(2):317-23. doi:10.7589/0090-3558-29.2.317.

Proulx G, Kolenosky AJ, Cole PJ, Drescher RL. 1995. A humane killing trap for lynx (Felis lynx): the Conibear $330^{\mathrm{mit}}$ with clamping bars. J Wildl Dis. 31(1):57-61. doi:10.7589/0090-3558-31.1.57.

Responsive Management (Harrisonburg, VA). 2015. Trap use, furbearers trapped, and trapper characteristics in the United States in 2015 Washington (DC): Association of Fish and Wildlife Agencies (US). Grant no.: F15AP00158. 